\title{
Retrospective Study of Canine Nasal Tumor Treated with Hypofractionated Radiotherapy
}

\author{
Takuya MARUO ${ }^{1)}$, Takuo SHIDA ${ }^{1,2) *}$, Yasuhiro FUKUYAMA ${ }^{1)}$, Soshi HOSAKA ${ }^{1)}$, Masashi NODA ${ }^{1)}$, Tetsuro ITO $^{1)}$, \\ Hiroki SUGIYAMA $^{1)}$, Takeshi ISHIKAWA ${ }^{1)}$ and Hiroo MADARAME ${ }^{1,2)}$ \\ 1) Veterinary Teaching Hospital and ${ }^{2}$ School of Veterinary Medicine, Azabu University, 1-17-71 Fuchinobe, Chuo, Sagamihara, \\ Kanagawa 252-5201, Japan
}

(Received 8 May 2010/Accepted 21 September 2010/Published online in J-STAGE 5 October 2010)

ABSTRACT. The object of this study was to evaluate hypofractionated multiportal field and two-portion (rostral and caudal portions divided by the eyelid) radiation therapy for canine nasal tumors. Sixty-three dogs underwent multiportal hypofractionated radiation therapy. The radiation field was divided into rostral and caudal portions by the eyelid. Treatments were performed four times for 57 dogs. The median irradiation dose/fraction was $8 \mathrm{~Gy}$ (range, 5-10 Gy); the median total dose was $32 \mathrm{~Gy}$ (10-40 Gy). Improvement of clinical symptoms was achieved in $53(84.1 \%)$ of 63 cases. Median survival time was 197 days (range, 2-1,080 days). Median survival times with and without destruction of the cribriform plate before radiotherapy were 163 and 219 days, respectively. There was no significant difference between them. No other factors were related to survival according to a univariate analysis. All radiation side effects, except one, were grade I according to the VRTOG classification. It was not necessary to treat any dogs for skin side effects. One dog (1.6\%) developed an oronasal fistula 1 year after completion of radiation therapy. This radiation protocol may be useful in reducing radiation side effects in dogs with cribriform plate destruction.

KEY WORDS: canine, hypofractionated radiotherapy, multiportal fields, nasal tumor, palliative.

J. Vet. Med. Sci. 73(2): 193-197, 2011

Tumors involving the nasal cavity and nearby sinuses are uncommon in dogs, and they account for approximately $1 \%$ of all reported canine tumors [29]. Because nasal tumors metastasize rarely, late in the course of disease, therapy is directed at controlling localized disease [19]. Without treatment, the median survival time of dogs has been reported to be $1.5-4.1$ months $[22,24,30]$. Surgery alone never prolongs survival time $[16,22]$, but it may also deteriorate quality of life. In regard to chemotherapy, Langova et al. reported the effectiveness of alternating doses of doxorubicin and carboplatin in conjunction with oral piroxicam [14], though their sample size was small [14]. Radiation therapy is the treatment of choice [29], and reported survival times have ranged from $7.4-47.7$ months $[1-3,8,11-13,17,20$, $21,23,27,28,30]$.

Various radiation treatment protocols in veterinary medicine have been reported. For total dose increasing, 19 [11] or 21 [5] fractions at $3 \mathrm{~Gy} /$ fraction over 1 month have been used. Because this method requires frequent anesthesia and is expensive, it is a heavy burden on the dog and owner. Hypofractionated radiotherapy has been used to address these issues [7, 18].

Hypofractionated radiation therapy results in late side effects that are more severe than with fractionated radiation therapy; blindness was reported following radiation treatment of dogs with nasal tumors [7, 18]. To address this, multiportal field and two-portions (rostral and caudal por-

\footnotetext{
* Correspondence to: Shida, T., Veterinary Teaching Hospital, Azabu University, 1-17-71 Fuchinobe, Chuo, agamihara, Kanagawa 252-5201, Japan.

e-mail: shida@azabu-u.ac.jp
}

tions divided by the eyelid) radiotherapy (Fig. 1) have been used to deliver the radiation dose to the tumor and achieve a decreased dose to surrounding tissue and, thus, prevent severe side effects, such as blindness and skin or bone necrosis, as previously reported in 18 cases [26].

The purpose of this study was to evaluate hypofractionated multiportal field and two-portion (rostral and caudal portions divided by the eyelid) radiation therapy for canine nasal tumors

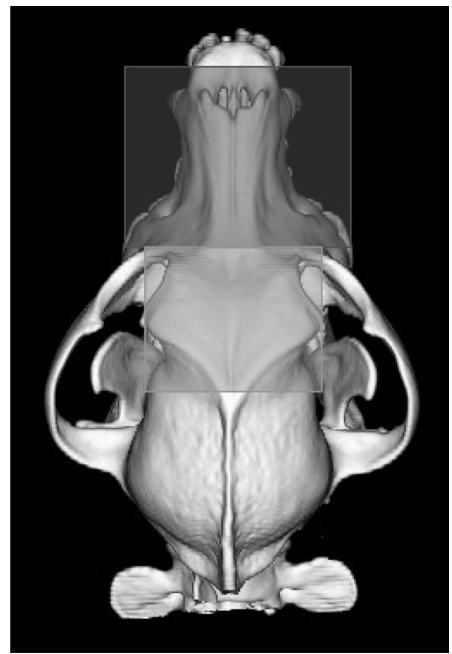

Fig. 1. To reduce the dose of the eye balls, the irradiation field was divided into two portions (rostral and caudal portions) by the eyelid. 


\section{MATERIALS AND METHODS}

A total of 63 dogs with nasal tumors were treated by radiation therapy between November 2005 and February 2009 at Azabu University Veterinary Teaching Hospital. Dogs treated by surgery were excluded.

Information obtained from the medical records or telephone interviews with the owner or referring veterinarian included breed, sex, age, body weight, clinical symptoms, duration of clinical symptoms from start to first visit, modified TNM classification, histopathology, radiation details (treatment number, portal number, irradiation dose/fraction, total dose, total dose of the center of the eyeball on the irradiated), response to treatment, posttreatment CT imaging findings, survival time, cause of death, and radiation side effects.

The tumors were staged based on clinical and CT findings using Adams's classification (a modification of the WHO system) [3]. Clinical efficacy was assessed after irradiation, and response was assessed by CT 1 month after radiation therapy was completed. Survival time was calculated from the completion of radiation treatment to the time of death. The toxicity criteria of the Veterinary Radiation Therapy Oncology Group (VRTOG) [10] were used to assess the radiation side effects.

For radiation therapy, a 4-MV linear accelerator (Mevatron, Toshiba, Tokyo, Japan) was used. Multiportal hypofractionated radiation therapy was performed once a week, with most of the dogs receiving four treatments. The radiation field was divided into rostral (Fig. 2, left) and caudal portions (Fig. 2, right) by the eyelid. The caudal portion was planned to avoid the orbit in order to reduce radiation side effects. We planned to irradiate the gross tumor with more than $80 \%$ of the isocenter dose. Treatment was assessed by CT 1 month after completion of radiotherapy.
Survival analysis was performed using the Kaplan-Meier method, and the log-rank test was used to assess survival time and factors related to outcome, including age, body weight, duration of clinical signs from onset to first visit, with or without facial deformity, and with or without cribriform plate destruction. The level of significance was set at $P<0.05$.

\section{RESULTS}

The common breeds included in the present study were the Shiba Inu $(n=8)$, Golden Retriever $(n=7)$, Labrador Retriever $(n=7)$, Shetland Sheepdog $(n=7)$, Pembroke Welsh Corgi $(n=5)$, Papillon $(n=3)$, Beagle $(n=3)$, Maltese $(n=3)$ and Miniature Dachshund $(n=2)$. The other breeds were the American Cocker Spaniel, Miniature Schnauzer, Toy Poodle, Siberian Husky, Border Collie, Alaskan Malamute $(n=1$ each $)$ and mixed breed $\operatorname{dog}(n=11)$. Overall, 35 dogs were male and 28 were female, the mean age was 10 years (range, 4-15 years) and the median body weight was $13.0 \mathrm{~kg}$ (range, $3.0-47.0 \mathrm{~kg}$ ). The clinical findings included epistaxis in 53 dogs $(84.1 \%)$, sneezing in 43 dogs (68.3\%), nasal discharge in 21 dogs $(33.3 \%)$, facial deformity in 29 dogs $(46 \%)$, exophthalmos in 10 dogs $(15.9 \%)$ and neurological symptoms in four dogs $(6.3 \%)$. The median duration of clinical signs from onset to first visit was 102 days (range, 8-1,080 days).

One dog had stage II disease, 17 dogs had stage III disease and 35 dogs had stage IV disease. There were five dogs (7.9\%) with regional lymph node metastases; none had lung metastases. The following tumor types were identified: adenocarcinoma $(29,46.0 \%)$, squamous cell carcinoma (10, $15.9 \%)$, osteosarcoma $(6,9.5 \%)$, undifferentiated carcinoma $(3,4.8 \%)$, transitional carcinoma $(3,4.8 \%)$, hemangiosarcoma $(2,3.2 \%)$, chondrosarcoma $(2,3.2 \%)$ and
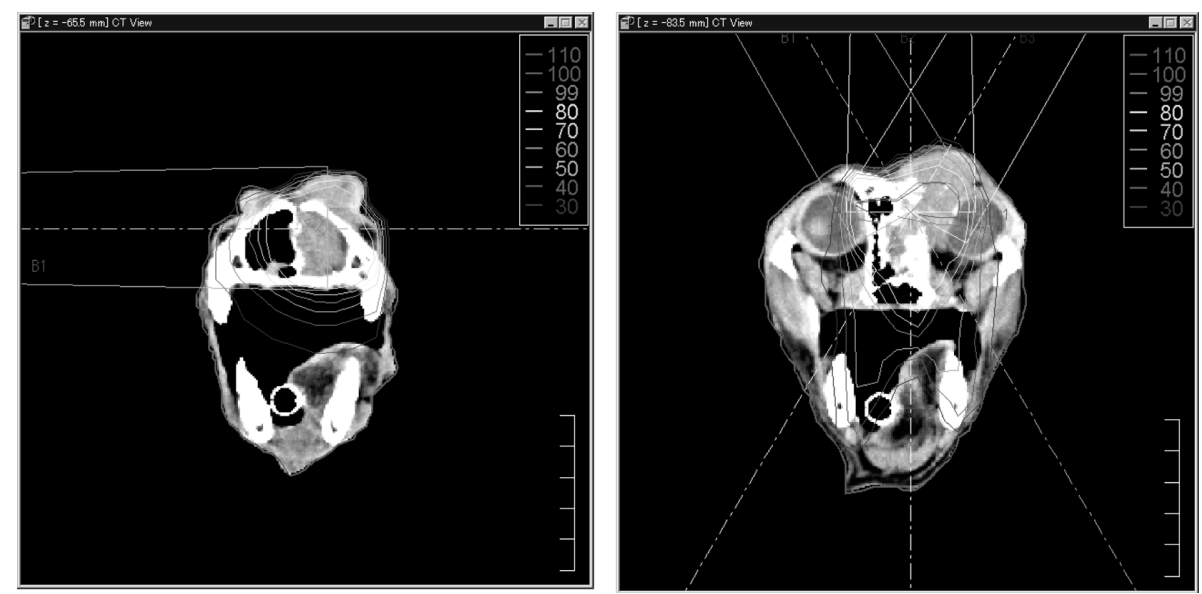

Fig. 2. Radiotherapy planning of the posterior portion by the eyelid. To reduce the radiation dose in the eye and other surrounding tissues, radiation was divided into three portal fields, and the tumor was irradiated with at least $80 \%$ of the isocenter dose (left and right: rostral and caudal portions, respectively). The median total dose of the center of the eyeball on the irradiated side was 9.5 Gy (range, $0-35.5 \mathrm{~Gy}$ ) for irradiation of the caudal portion in 55 of the dogs. 
undifferentiated tumor $(8,12.7 \%)$.

Treatment was performed four times in $57 \mathrm{dogs}$, three times in three dogs and one, two and five times in one dog each. Portal number of rostral portion was rotational irradiation 45 dogs, 4 portal irradiation 12 dogs, and a single portal irradiation $1 \mathrm{dog}$ in 58 dogs. Portal number of caudal portion was all 3 portal irradiation in 55 dogs. Furthermore, three dogs had tumors that had progressed to the caudal part of the frontal sinus and were irradiated from the caudal part to caudal portion by opposing portal irradiation. The median irradiation dose/fraction was 8 Gy (range, 5-10 Gy), and the median total dose was 32 Gy (10-40 Gy); the median total dose of the center of the eyeball on the irradiated side was 9.5 Gy (range, 0-35.5 Gy) for irradiation of the caudal portion in 55 of the dogs.

Improvement of clinical symptoms was achieved in 53 $(84.1 \%)$ of the 63 cases. CT images at 1 month after completion of radiotherapy were available in 30 cases, and the result was complete regression in three cases $(10.0 \%)$, partial regression in 16 cases $(53.3 \%)$, stable disease in 10 cases $(33.3 \%)$ and progressive disease in one case $(3.3 \%)$. The median survival of all dogs was 197 days (range, 2-1,080 days). The 1- and 2-year survival rates were 25.4 and $7.9 \%$, respectively. Death was due to local disease in 37 dogs $(90.2 \%)$ and disease other than nasal cancer in four dogs $(9.8 \%)$.

Acute and late radiation side effects affected the skin and eyes. All except the late side effects were grade I according to the VRTOG classification: 15 dogs $(23.8 \%)$ had hair loss, 11 dogs (17.5\%) had color change and 13 dogs (20.6\%) had conjunctivitis. No treatment was needed for skin side effects. One year later, an oronasal fistula occurred after completion of radiation therapy in one $\operatorname{dog}(1.6 \%)$.

No factors were related to survival according to a univariate analysis. The dogs were divided into two groups by median duration of clinical signs from onset to first visit (102 days). There was no significant difference between 102 days and $<102$ days, with median survival times of 296 and 197 days, respectively. There was no difference in median survival time between dogs with $(n=29,150$ days) and those without facial deformity $(n=34,234$ days). Similarly, there was no difference in median survival time between dogs with cribriform plate destruction before radiotherapy ( $n=35,163$ days) and those without $(n=28,219$ days; Fig. 3). Furthermore, there were no significant differences by age, body weight and radiation side effects.

\section{DISCUSSION}

In this study, hypofractionated radiotherapy was effective for nasal tumors and resulted in mild radiation acute side effects. Improvement of clinical symptoms was achieved in $53(84.1 \%)$ of 63 cases. CT images 1 month after completion of radiotherapy were available in 30 cases, and the result was complete regression in three cases $(10.0 \%)$ and partial regression in 16 cases $(53.3 \%)$. The median survival of all dogs was 197 days (range, 2-1,080 days). All side

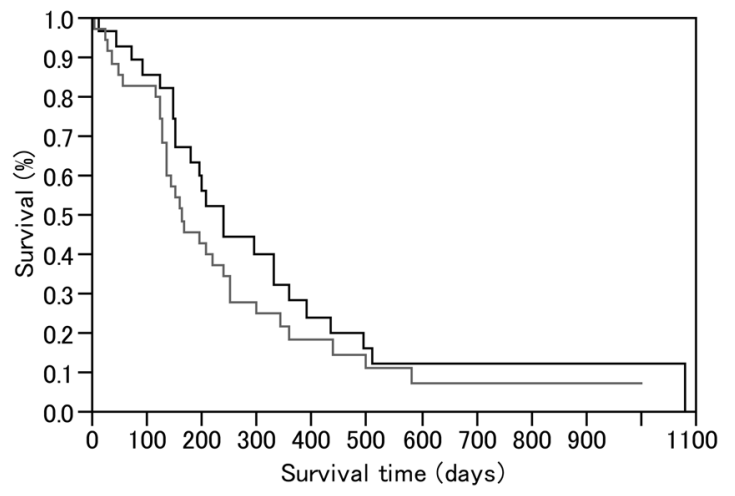

Fig. 3. Kaplan-Meier survival curves with or without cribriform destruction. There was no difference in median survival time between dogs with cribriform plate destruction before radiotherapy (gray line, $n=35,163$ days) and those without (balck line, $n=28,219$ days).

effects, except for late ones, were grade I according to the VRTOG classification, and no dogs required treatment for skin side effects.

Mellanby et al. [18] reported that a retrospective study was undertaken on 56 dogs treated for nasal tumors by megavoltage radiotherapy with a hypofractionated schedule consisting of four doses of 9 Gy given at intervals of seven days. The median survival time in their study was 212 days. Gieger et al. [7] reported data from 48 dogs with nasal carcinomas treated with hypofractionated radiation therapy due to the extent of local disease or concurrent disease (40\%) and the owners requesting palliative radiotherapy because of concerns about cost, potential for toxicity or travel/hospitalization associated with definitive radiation therapy. The total radiation dose ranged from 16 to 40 Gy (median 24 $\mathrm{Gy}$ ), and the dose per fraction ranged from 4 to $10 \mathrm{~Gy}$ (median 8 Gy). The median survival time in their study was 146 days. The median survival time of 197 days in the present study does not differ greatly from either of those reports. However, a more fractionated protocol produces a better therapeutic gain than a hypofractionated protocol. Previous studies reported median survival times of 8.9-19.7 months with 10-19 fractions $[1,2,11,17,27]$. In the present study, the median survival time was shorter than that in previous studies because of the lower total irradiation dose, as is found with other hypofractionated protocols.

Advanced disease is believed to be associated with a poor prognosis. The reported prognostic factors are destruction of the cribriform plate [1], tumor type [1, 27], tumor stage $[1,3,4,9,11,27]$, age $>10$ years [11], regional lymph node or pulmonary metastasis [8], resolution of clinical signs after treatment [7, 23], tumor regression [28], facial deformity [23], three or more treatments/week [30] and a cumulative minimum tumor dose of at least $37 \mathrm{~Gy}$ [30]. In regard to the stage in particular, various stage classifications have been reported, and lower stages resulted in longer survival than higher stages $[1,3,4,9,11,27]$. However, the present 
results and other hypofractionated protocols $[7,18]$ also showed no significant difference between tumor stage and survival time; thus, it was thought that the hypofractionated protocol was inappropriate for early stages. However, a more fractionated protocol for dogs with destruction of the cribriform plate by CT resulted in median survival times of 6.7 months [1] and 6.6 months [9] (the tumors were classified in stage IV together). The median survival times for stage I-III tumors were 23.1, 14.0 and 15.7 months [1], respectively, and that of the latter stage III tumors was 15.1 months [9]. These survival times resembled our results for dogs with destruction of the cribriform plate. Thus, for dogs with destruction of the cribriform plate, the current protocol was useful, as it reduced radiation side effects and required less frequent treatments.

Local tumor control can be achieved with an adequate margin; generally, the larger the radiation field is, the more serious the radiation side effects are [6]. Early side effects of radiation therapy are generally self-limiting, and recovery is rapid [15]. Therefore, even if severe side effects are predicted, it is important to plan an adequate margin to control the tumor. Concerning the radiation side effects of fractionated protocols, McEntee et al. [17] utilized an irradiation dose of 41.8-54 Gy and documented transient conjunctivitis $(67 \%)$ and oral mucositis (81\%). Significant ophthalmic complications in $48 \%$ of the cases. Adams et al. [2] utilized an irradiation dose of $42 \mathrm{~Gy}$, and 15 out of 53 dogs lost sight in 1 (11 dogs) or both (4) eyes following radiotherapy. Roberts et al. [25] reported ophthalmic complications following megavoltage irradiation of the nasal and paranasal cavities in dogs with a median total dose was 40 Gy (range, 36.8-50 Gy), and the ocular complications were classified as mild $(17.2 \%)$ and severe $(58.6 \%)$. On the other hand, by hypofractionated protocols, Mellanby et al. [18] reported that $57 \%$ of the dogs required treatment for acute side effects. No long-term side effects were observed, apart from in one dog, which became blind as a result of keratitis [18]. Gieger et al. [7] reported that chronic ocular toxicities were reported $13 \%$ of the dogs that had at least one eye irradiated [7]. Chronic toxicities included conjunctivitis, corneal ulceration or perforation, loss of vision, keratoconjunctivities sicca, severe mucoid ocular discharge, cataract formation, blepharospasm and uveitis [7]. The radiation side effects following nasal tumor treatment have included severe symptoms related to the orbit $[2,3,12,17,21,23,25$, 27]. Serious radiation side effects and frequent treatment are of little benefit for dogs with high stage tumors because the treatment for nasal tumors is palliation, not radical cure. Thus, a once-a-week, four-treatment hypofraction protocol was used to reduce frequent anesthesia, and multiportal fields and two portions (rostral and caudal portions divided by the eyelid) were planned to reduce the exposure of the surrounding normal tissues and the orbit to radiation. In our results, the median total dose of the center of the eyeball was low, $9.5 \mathrm{~Gy}$; therefore the radiation side effects were thought to be mild.

The once-a-week, multiportal field, two-portion protocol (rostral and caudal portions divided by the eyelid) with four hypofractions was useful to reduce the number of treatments, save cost and reduce the burden of the dogs and their owners because the majority of cases experienced amelioration of clinical symptoms, and it was not necessary to treat radiation side effects in dogs with cribriform plate destruction.

\section{REFERENCES}

1. Adams, W. M., Kleiter, M. M., Thrall, D. E., Klauer, J. M., Forrest, L. J., La Due, T. A. and Havighurst, T. C. 2009. Prognostic significance of tumor histology and computed tomographic staging for radiation treatment response of canine nasal tumors. Vet. Radiol. Ultrasound 50: 330-335.

2. Adams, W. M., Bjorling, D. E., McAnulty, J. E., Green, E. M., Forrest, L. J. and Vail, D. M. 2005. Outcome of accelerated radiotherapy alone or accelerated radiotherapy followed by exenteration of the nasal cavity in dogs with intranasal neoplasia: 53 cases (1990-2002). J. Am. Vet. Med. Assoc. 227: 936941.

3. Adams, W. M., Miller, P. E., Vail, D. M., Forrest, L. J. and MacEwen, E. G. 1998. An accelerated rechnique for irradiation of malignant canine nasal and paranasal sinus tumors. Vet. Radiol. Ultrasound 39: 475-481.

4. Buchholz, J., Hagen, R., Leo, C., Ebling, A., Roos, M., KaserHotz, B. and Bley, C. R. 2009. 3D conformal radiation therapy for palliative treatment of canine nasal tumors. Vet. Radiol. Ultrasound 50: 679-683.

5. Correa, S. S., Mauldin, G. N., Mauldin, G. E. and Patnaik, A. K. 2003. Efficacy of cobalt-60 radiation therapy for the treatment of nasal cavity nonkeratinizing squamous cell carcinoma in the dog. J. Am. Anim. Hosp. Assoc. 39: 86-89.

6. Emami, B., Lyman, J., Brown, A., Coia, L., Goitein, M., Munzenrider, J. E., Shank, B., Solin, L. J. and Wesson, M. 1991. Tolerance of normal tissue to therapeutic irradiation. Int. J. Radiat. Oncol. Biol. Phys. 21: 109-122.

7. Gieger, T., Rassnick, K., Siegel, S., Proulx, D., Bergman, P., Anderson, C., LaDue, T., Smith, A., Northrup, N. and Roberts, R. 2008. Palliation of clinical signs in 48 dogs with nasal carcinomas treated with coarse-fraction radiation therapy. J. Am. Anim. Hosp. Assoc. 44: 116-123.

8. Henry, C. J., Brewer, W. G. Jr., Tyler, J. W., Brawner, W. R., Henderson, R. A., Hankes, G. H. and Royer, N. 1998. Survival in dogs with nasal adenocarcinoma: 64 cases (1981-1995). J. Vet. Intern. Med. 12: 436-439.

9. Kondo, Y., Matsunaga, S., Mochizuki, M., Kadosawa, T., Nakagawa, T., Nishimura, R. and Sasaki, N. 2008. Prognosis of canine patients with nasal tumors according to modified clinical stages based on computed tomography: a retrospective study. J. Vet. Med. Sci. 70: 207-212.

10. LaDue, T. and Klein, M. K. 2001. Toxicity criteria of the veterinary radiation therapy oncology group. Vet. Radiol. Ultrasound 42: 475-476.

11. LaDue, T. A., Dodge, R., Page, R. L., Price, G. S., Hauck, M. L. and Thrall, D. E. 1999. Factors influencing survival after radiotherapy of nasal tumors in $130 \mathrm{dogs}$. Vet. Radiol. Ultrasound 40: 312-317.

12. Lana, S. E., Dernell, W. S., Lafferty, M. H., Withrow, S. J. and Larue, S. M. 2004. Use of radiation and a slow-release cisplatin formulation for treatment of canine nasal tumors. Vet. Radiol. Ultrasound 45: 577-581. 
13. Lana, S. E., Dernell, W. S., LaRue, S. M., Lafferty, M. J., Douple, E. B., Brekke, J. H. and Withrow, S. J. 1997. Slow release cisplatin combined with radiation for the treatment of canine nasal tumors. Vet. Radiol. Ultrasound 38: 474-478.

14. Langova, V., Mutsaers, A. J., Phillips, B. and Straw, R. 2004. Treatment of eight dogs with nasal tumours with alternating doses of doxofubicin and carboplatin in conjunction with piroxicam. Aust. Vet. J. 82: 676-680.

15. LaRue, S. M. and Gillette, E. L. 2007. Radiation Therapy. pp. 193-210. In: Small Animal Clinical Oncology, 4th ed. (Withrow, S. J. and Vail, D. M. eds.), Saunders, Philadelphia.

16. MacEwen, G., Withrow, S. J. and Patnaik, A. K. 1977. Nasal tumors in the dogs: retrospective evaluation of diagnosis, prognosis, and treatment. J. Am. Vet. Med. Assoc. 170: 45-48.

17. McEntee, M. C., Page, R. L., Heidner, G. L., Cline, J. M. and Thrall, D. E. 1991. A retrospective study of 27 dogs with intranasal neoplasms treated with cobalt radiation. Vet. Radiol. Ultrasound 32: 135-139.

18. Mellanby, R. J., Stevenson, R. K., Herrtage, M. E., White, R. A. S. and Dobson, J. M. 2002. Long-term outcome of 56 dogs with nasal tumours treated with four doses of radiation at intervals of seven days. Vet. Rec. 151: 253-257.

19. Moore, A. S. and Ogilvie, G. K. 2006. Tumors of the respiratory tract. pp. 405-419. In: Managing the canine cancer patient (Ogilvie, G. K. and Moore, A. S. eds.). Veterinary Learning Systems, Yardley.

20. Morris, J. S., Dunn, K. J., Dobson, J. M. and White, R. A. S. 1994. Effects of radiotherapy alone and surgery and radiotherapy on survival of dogs with nasal tumours. J. Small Anim. Pract. 35: 567-573.

21. Nadeau, M. E., Kitchell, B. E., Rooks, R. L. and LaRue, S. M. 2004. Cobalt radiation with or without low-dose cisplatin for treatment of canine naso-sinus carcinomas. Vet. Radiol. Ultrasound 45: $362-367$.

22. Norris, A. M. 1979. Intranasal neoplasms in the dog. J. Am. Anim. Hosp. Assoc. 15: 231-236.
23. Northrup, N. C., Etue, S. M., Ruslander, D. M., Rassnick, K. M., Hutto, D. L., Bengtson, A., Rand, W. and Moore, A. S. 2001. Retrospective study of orthovoltage radiation therapy for nasal tumors in 42 dogs. J. Vet. Intern. Med. 15: 183-189.

24. Rassnick, K. M., Goldkamp, C. E., Erb, H. N., Scrivani, P. V., Njaa, B. L., Gieger, T. L., Turek, M. M., McNiel, E. A., Proulx, D. R., Chun, R., Mauldin, G. E., Phillips, B. S. and Kristal, O. 2006. Evaluation of factors associated with survival in dogs with untreated nasal carcinomas: 139 cases (1993-2003). J. Am. Vet. Med. Assoc. 229: 401-406.

25. Roberts, S. M., Lavach, J. D., Severin, G. A., Withrow, S. J. and Gillette, E. J. 1987. Ophthalmic complications following megavoltage irradiation of the nasal and paranasal cavities in dogs. J. Am. Vet. Med. Assoc. 190: 43-47.

26. Shida, T., Maruo, T., Fukuyama, Y., Mori, A., Kawamura, H., Takeda, H., Takahira, A., Inoue, A., Yamada, T., Ito, T., Madarame, H., Kayanuma, H. and Suganuma, T. 2008. The effects of high-dosed, hypofractionated, multi-field radial irradiation to dogs with malignant nasal tumor. J. Anim. Clin. Med. 17: 1-6 (in Japanese).

27. Théon, P., Madewell, B. R., Harb, M. F. and Dungworth, D. L. 1993. Megavoltage irradiation of neoplasms of the nasal and paranasal cavities in 77 dogs. J. Am. Vet. Med. Assoc. 202: 1469-1475.

28. Thrall, D. E., Heidner, G. L., Novotney, C. A., McEntee, M. C. and Page, R. L. 1993. Failure patterns following cobalt irradiation in dogs with nasal carcinoma. Vet. Radiol. Ultrasound 34: 126-133.

29. Turek, M. M. and Lana, S. E. 2007. Canine nasosinal tumors. pp. 525-539. In: Small Animal Clinical Oncology (Withrow, S. J. and Vail, D. M. eds.), Saunders Elsevier, St. Louis.

30. Yoon, J. H., Feeney, D. A., Jessen, C. R. and Walter, P. A. 2008. External-beam Co-60 radiotherapy for canine nasal tumors: a comparison of survival by treatment protocol. Res. Vet. Sci. 84: 140-149. 